\title{
Discrimination training and reversal in groups of honey bees'
}

GORDON BERMANT AND NORMAN E. GARY

UNIVERSITY OF CALIFORNIA, DAVIS

Worker bees were given the opportunity to forage for sugar syrup placed at the end of a runway attached to their hive. After stable foraging activity had developed, the collection of syrup was made contingent upon the correct response to a simultaneous color discrimination problem. Bees that foraged regularly learned the discrimination within several hours. Correct performance fell to a low level immediately following stimulus reversal, then improved rapidly.

Since the early, now classic work of von Frisch and his colleagues on navigation and intra-specific communication, the behavior of honey bees has aroused the intense interest of many zoologists and animal psychologists. The elegance of the communication system, particularly in view of its apparently unlearned basis, 2 has made it the standard example of the highest level of invertebrate adaptation. Investigations of bees' capacity for plasticity, however, are relatively scarce (Johnson \& Wenner, 1965; Weiss, 1953; Wenner \& Johnson, 1965).

A method recently has been developed (Gary \& Bermant, 1966) which permits the close observation of bees in various types of learning or conditioning situations with minimal dismuption or alteration of normal hive conditions. ${ }^{3}$ In this report we present the results of experiments on a simultaneous color discrimination and reversal problem.

Method

A glass-walled observation hive $(100 \times 48 \times 6.5 \mathrm{~cm})$ containing approximately 10,000 individuals was maintained in the experimental room. There were two exits from the hive. One of these Ied directly outdoors; this outdoor exit permitted the colony to forage under normal conditions. The other exit led onto a Plexiglas-floored runway $(2 \times 18 \times 140 \mathrm{~cm})$ in the experimental room.

When the hive was first opened to the runway, several animals entered and explored the closer portions. Bees were encouraged to use the entire runway by making peppermint-scented sugar syrup available at positions progressively farther away from the hive entrance. In this way a steady progression of workers to and from the end of the runway was developed. For each experiment, 40-100 animals were picked at random from the foraging group and anesthetized with carbon dioxide (10 sec.) so that identification tags could be glued onto their thoraxes.

Discrimination training was effected under the following conditions: the last $25 \mathrm{~cm}$ of the runway were divided longitudinally in half by a white cardboard barrier. The entrance to each side of the divided runway consisted of a colored card provided with a passageway. The cards served as discriminanda; one was yellow, and the other was blue. Another set of colored passageways was placed $10 \mathrm{~cm}$ farther down the divided runway.

Each of the two feeding compartments $(9 \times 15 \mathrm{~cm})$ at the end of the runway contained a liquid feeder identical to that used to establish foraging. The feeder associated with the correct colored card contained the standard peppermint-sugar syrup. The other feeder contained a $10 \% \mathrm{NaCl}$ solution; workers did not ingest this solution more than once.

For a given experiment the initially correct color, blue or yellow, was chosen arbitrarily. Trial duration was $15 \mathrm{~min}$. The positions of the discriminanda and feeders were reversed after each trial to control for position preferences.

A color discrimination reversal task was instituted after the regularly responding foragers had reached a high level of correct performance on the initial problem. Reversal procedure consisted of changing the positions of the cards once without moving the feeders.

During an experiment the animals were observed for 6-7 hr. per day, on a schedule of $45 \mathrm{~min}$. on, $15 \mathrm{~min}$. off. At the end of a day's testing the discriminanda and partition were removed and a single colorless sugar

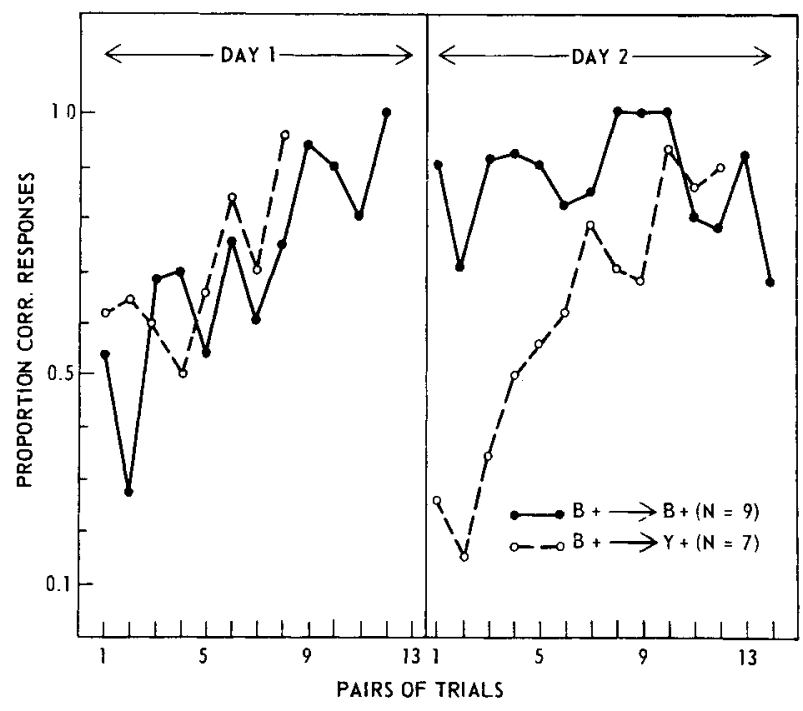

Fig. 1. Solid line: demonstration of retention following an overnight pause. Dotted line: the effect of stimulus reversal following an overnight pause. 


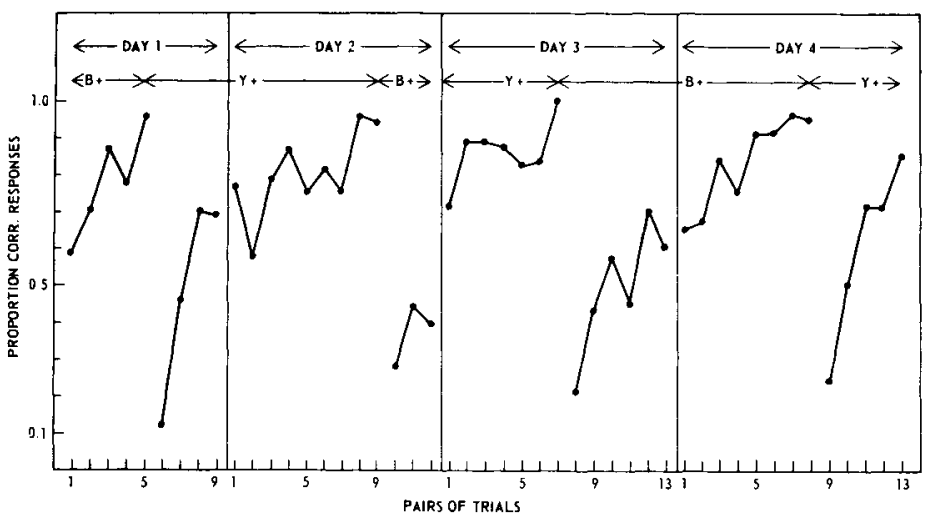

Fig. 2. The effects of five reversals.

syrup container was placed in the center of the end of the runway.

Three response types were recorded: (1) correct, in which a bee entered the correct feeding compartment, drank, then returned to the hive; (2) incorrect, in which the bee entered the incorrect feeding compartment, then returned to the hive; and (3) incorrectcorrect, in which the bee first entered the incorrect feeding compartment, then left it and went directly to the correct compartment, drank, and then returned to the hive. The measure of performance chosen for analysis was the proportion of correct responses in pairs of trials, i.e.,

$\frac{\text { correct }}{\text { correct }+ \text { incorrect }+ \text { incorrect-correct }}$

Only those animals that made at least one response $(1,2$, or 3$)$ on at least $50 \%$ of the trials were included in the final analysis of each experiment.

\section{Results}

The solid line in Fig. 1 represents the performance of a group that was trained for two successive days with blue positive; the dotted line shows the responses of a group that was reversed from $\mathrm{B}+$ to $\mathrm{Y}+$ on day 2 . The effects of reversal were obvious: the bees in the reversal group did not reach $50 \%$ correct performance on day 2 until trials 7-8. They reached $90 \%$ correct on trials 19-20.

Figure 2 displays the responses of nine bees over four days; during that time five reversals were administered. Four of these were carried out during the day, and one was performed overnight following sixtrials on the previously correct color. When reversal occurred during the day, correct performance fell to $12-28 \%$ on the first few trials, then climbed rapidly. In the two cases where the same color remained correct after the overnight pause, initial correct performance was approximately the same as it had been on the terminal trials of the previous day. The interpolation of six $B+$ trials at the end of day 2 had little effect on $\mathrm{Y}+$ performance during the morning of day 3 .

\section{Discussion}

These data confirm the ability of worker honey bees to learn simple discrimination problems under laboratory conditions; they also demonstrate a large but shortlived effect of stimulus reversal. We were unable to confirm the bee's potential for discrimination reversal improvement; the short duration of foraging life in the runway did not allow a sufficiently large number of reversals to be made.

\section{References}

Gary, N., \& Bermant, G. A system for investigating the foraging behavior of honey bees, Apis mellifera L., in the laboratory. (Submitted to J. apicult. Res.)

Johnson, D., \& Wenner, A. A relationship between conditioning and communication in honey bees. Amer. Zoologist, 1965, 5 , 653 (Abstract).

Weiss, K. v. Versuche mit Bienen und Wespen in farbigen Labyrinthen. Z. f. Tierpsychol., 1953, 10, 29-44.

Wenner, A., \& Johnson, D. Mass conditioning of honey bees in the laboratory, Amer. Zoologist, 1965, 5, 714 (Abstract).

\section{Notes}

1. Research supported by NSF Grant GB-3328 and University of California Research Grant No. D-247.

2. The recent investigations of Wenner and his colleagues (see bibliography) indicate that the extent to which communication about food source location depends on innate responses may be less than previously believed.

3. A similar technique was apparently developed independently by Butler, and later modified by Wenner \& Johnson (1965). Complete technical specifications for the present apparatus are presented in Gary \& Bermant (1966). 